\title{
THE DEEP BLUE COLOR OF HD 189733b: ALBEDO MEASUREMENTS WITH HUBBLE SPACE TELESCOPE/SPACE TELESCOPE IMAGING SPECTROGRAPH AT VISIBLE WAVELENGTHS
}

\author{
Thomas M. Evans ${ }^{1}$, Frédéric Pont ${ }^{2}$, David K. Sing ${ }^{2}$, Suzanne Aigrain ${ }^{1}$, Joanna K. Barstow ${ }^{1}$, Jean-Michel Désert ${ }^{3,7}$, \\ Neale Gibson $^{4}$, Kevin Heng $^{5}$, Heather A. Knutson ${ }^{3}$, and Alain Lecavelier des Etangs ${ }^{6}$ \\ ${ }^{1}$ Department of Physics, University of Oxford, Denys Wilkinson Building, Keble Road, Oxford OX1 3RH, UK; tom.evans@astro.ox.ac.uk \\ ${ }^{2}$ School of Physics, University of Exeter, EX4 4QL Exeter, UK \\ ${ }^{3}$ Division of Geological and Planetary Sciences, California Institute of Technology, Pasadena, CA 91125, USA \\ ${ }^{4}$ European Southern Observatory, Karl-Schwarzschild-Strasse 2, D-85748 Garching, Germany \\ ${ }^{5}$ University of Bern, Center for Space and Habitability, Sidlerstrasse 5, CH-3012 Bern, Switzerland \\ ${ }^{6}$ Institut d'Astrophysique de Paris, UMR7095 CNRS, Universite Pierre et Marie Curie, 98 bis Boulevard Arago, F-75014 Paris, France \\ Received 2013 May 31; accepted 2013 June 15; published 2013 July 11
}

\begin{abstract}
We present a secondary eclipse observation for the hot Jupiter HD $189733 \mathrm{~b}$ across the wavelength range $290-570 \mathrm{~nm}$ made using the Space Telescope Imaging Spectrograph on the Hubble Space Telescope. We measure geometric albedos of $A_{g}=0.40 \pm 0.12$ across $290-450 \mathrm{~nm}$ and $A_{g}<0.12$ across $450-570 \mathrm{~nm}$ at $1 \sigma$ confidence. The albedo decrease toward longer wavelengths is also apparent when using six wavelength bins over the same wavelength range. This can be interpreted as evidence for optically thick reflective clouds on the dayside hemisphere with sodium absorption suppressing the scattered light signal beyond $\sim 450 \mathrm{~nm}$. Our best-fit albedo values imply that HD 189733b would appear a deep blue color at visible wavelengths.
\end{abstract}

Key words: planets and satellites: atmospheres - stars: individual (HD 189733) - techniques: photometric

Online-only material: color figure

\section{INTRODUCTION}

The wavelength-dependent manner in which a planetary atmosphere reflects incident starlight reveals valuable details about its structure and composition. In this Letter, we present albedo measurements for the transiting hot Jupiter HD 189733b across the wavelength range $\lambda=290-570 \mathrm{~nm}$.

At these wavelengths, clear atmosphere models (i.e., without clouds) predict that hot Jupiter albedos are suppressed by alkali absorption (Sudarsky et al. 2000; Burrows et al. 2008). Observations to date have been largely consistent with these expectations (e.g., Rowe et al. 2008; Alonso et al. 2009; Snellen et al. 2009; Alonso et al. 2010; Snellen et al. 2010; Christiansen et al. 2010; Welsh et al. 2010; Désert et al. 2011; Kipping \& Spiegel 2011; Morris et al. 2013). Models also predict, however, that silicates and iron could condense in the uppermost layers of some atmospheres, raising the albedo significantly (Marley et al. 1999; Sudarsky et al. 2000). For instance, reflective clouds seem necessary to explain the relatively high albedo of Kepler7b (Kipping \& Bakos 2011; Demory et al. 2011).

By measuring the reflection signal of HD 189733b, our goal was to gauge the role of clouds/hazes ${ }^{8}$ in the atmosphere of this well-studied hot Jupiter. Motivation came from the observed transmission spectrum, which slopes downward from $290 \mathrm{~nm}$ out to $1 \mu \mathrm{m}$ (Pont et al. 2008; Sing et al. 2011) and possibly further into the infrared (Sing et al. 2009; Gibson et al. 2012a). A likely explanation for this feature is Rayleigh scattering by a high altitude haze of dust (Lecavelier Des Etangs et al. 2008). Furthermore, Huitson et al. (2012) detected the narrow core of the $\mathrm{Na} 589 \mathrm{~nm}$ doublet in transmission, but not the pressurebroadened wings that would be expected in a clear atmosphere (e.g., Fortney et al. 2010). Indirect evidence of clouds on the

\footnotetext{
7 Sagan Postdoctoral Fellow.

8 In this Letter, we use the words "cloud" and "haze" interchangeably as referring to any suspended condensates in the atmosphere.
}

dayside hemisphere has also been identified by Pont et al. (2013), who speculated that the albedo of HD 189733b might be high as a result.

In practice, we measured the reflection signal by monitoring the change in brightness of the star-planet system that occurred during secondary eclipse. Unlike the primary transit, which allows us to probe the day-night terminator region of the atmosphere, the secondary eclipse signal is directly related to the brightness of the dayside hemisphere. Although secondary eclipses have been measured previously for HD 189733b at infrared wavelengths (Deming et al. 2006; Knutson et al. 2007; Grillmair et al. 2008; Charbonneau et al. 2008; Agol et al. 2010; Knutson et al. 2012), our new observation constitutes the first measured for HD 189733b at short wavelengths, where thermal emission from the planet is negligible. Any detected light is therefore entirely due to scattering by the atmosphere. This allows us to place unambiguous constraints on the geometric albedo $A_{g}$, according to:

$$
A_{g}=\delta\left[\rho \frac{R_{\star}}{a}\right]^{-2}
$$

where $\delta$ is the fractional eclipse depth, $\rho=R_{p} / R_{\star}$ is the ratio of the planet and star radii, and $a$ is the orbital semimajor axis. Equation (1) follows from the formal definition of $A_{g}$, namely, the observed flux of scattered light at full phase divided by the flux that an isotropically scattering disk with the same crosssectional area would have if it were placed at the same location as the planet (e.g., Seager 2010).

\section{OBSERVATIONS AND DATA REDUCTION}

One secondary eclipse of HD 189733b was observed over four Hubble Space Telescope (HST) orbits using the Space Telescope Imaging Spectrograph (STIS) G430L grating (290-570 nm) for programme GO-13006 (PI F. Pont) on 2012 December 20. 


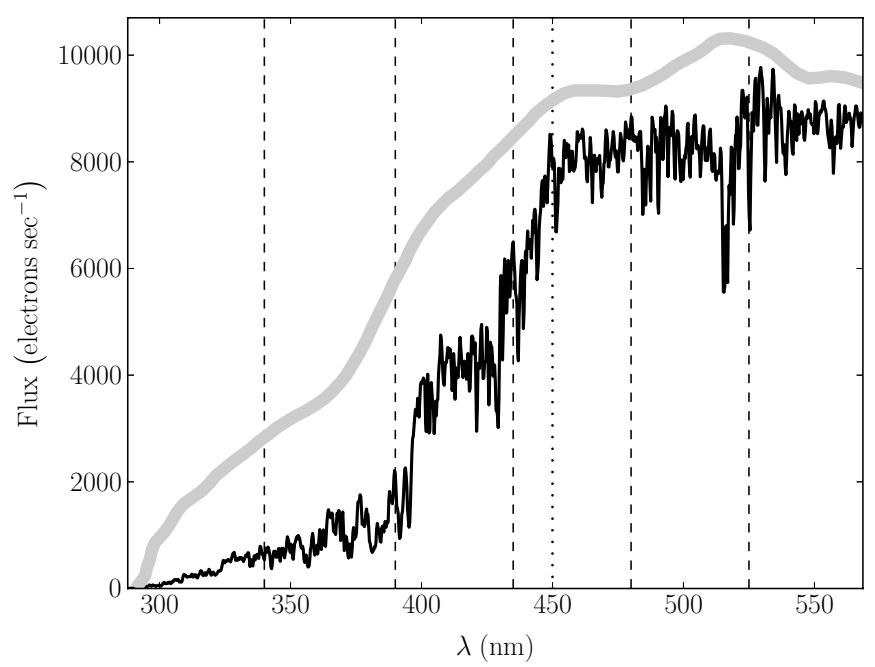

Figure 1. Mean system spectrum constructed from the out-of-eclipse spectra (solid black line) and G430L sensitivity curve with arbitrary normalization (thick gray line). Vertical lines indicate boundaries between the two-channel (dotted line) and six-channel (dashed lines) wavelength bins used for the lightcurve analysis.

Spectra taken during the first orbit exhibited much larger systematics than those taken in subsequent orbits due to settling of the telescope into its new pointing position and were not included in the analysis. Of the remaining three orbits, the first and third sampled the out-of-eclipse flux while the planet was close to full phase (star plus planet), and the second sampled the in-eclipse flux while the planet was fully obscured by the star (star only).

We used a wide $52^{\prime \prime} \times 2^{\prime \prime}$ slit to minimize time-varying slit losses caused by pointing drifts and reduced overheads by reading out only the $1024 \times 128$ pixel subarray containing the target spectrum. Based on previous experience with $H S T /$ STIS observations, we expected the first exposure of each satellite orbit to have systematically lower counts than the exposures immediately following. For this reason, a dummy $1 \mathrm{~s}$ exposure was taken at the beginning of each orbit, followed by a series of 35 science exposures with integration times of $64 \mathrm{~s}$. Unfortunately, the first science exposure of each satellite orbit still exhibited systematically lower flux levels, so we discarded these as well. Our final dataset thus consisted of 102 spectra taken over 237 minutes.

Images were reduced using the CALSTIS v2.40 pipeline and cleaned for cosmic rays. The spectra were then extracted using the IRAF apall routine with a 13 pixel-wide aperture. Background subtraction was not performed, as the background contribution was negligible. Spectra were Doppler-corrected to the heliocentric rest frame, corresponding to shifts of $\sim 1$ pixel along the dispersion axis. We generated photometric time series by integrating the flux from each individual exposure across the dispersion axis within different wavelength bins. Separate analyses were performed for a two-channel and six-channel binning: Figure 1 shows the mean system spectrum with adopted wavelength bins overplotted and Table 1 explicitly lists the wavelength ranges.

\section{LIGHTCURVE ANALYSIS}

The top panels of Figure 2 show the raw lightcurves for the two-channel binning. Within each orbit, the measured flux is dominated by an approximately repeatable decrease of
Table 1

Visible Albedo Measurements for HD 189733b

\begin{tabular}{lccc}
\hline \hline $\begin{array}{l}\Delta \lambda \\
(\mathrm{nm})\end{array}$ & $\begin{array}{c}\lambda_{c} \\
(\mathrm{~nm})\end{array}$ & $\begin{array}{c}\delta \\
(\mathrm{ppm})\end{array}$ & $A_{g}$ \\
\hline $290-450$ & 413 & $126_{-36}^{+37}$ & $0.40_{-0.11}^{+0.12}$ \\
$450-570$ & 510 & $1_{-30}^{+37}$ & $0.00_{-0.10}^{+0.12}$ \\
$290-340$ & 325 & $142_{-175}^{+176}$ & $0.45_{-0.55}^{+0.55}$ \\
$340-390$ & 368 & $123_{-87}^{+86}$ & $0.39_{-0.27}^{+0.27}$ \\
$390-435$ & 416 & $102_{-48}^{+48}$ & $0.32_{-0.15}^{+0.15}$ \\
$435-480$ & 459 & $53_{-36}^{+37}$ & $0.17_{-0.11}^{+0.12}$ \\
$480-525$ & 502 & $-35_{-36}^{+45}$ & $-0.11_{-0.11}^{+0.14}$ \\
$525-570$ & 547 & $7_{-36}^{+43}$ & $0.02_{-0.12}^{+0.14}$ \\
\hline
\end{tabular}

Notes. $\Delta \lambda$ and $\lambda_{c}$ are, respectively, the wavelength range and flux-weighted central wavelength for each channel. Uncertainties for $A_{g}$ have been propagated in quadrature.

1500-3000 ppm. Smaller amplitude correlations on shorter time scales are also evident, as well as a longer term decrease in the baseline flux level. Similar systematics are observed for the six-channel lightcurves, and are believed to be primarily caused by the thermal cycle of the satellite and the drift of the spectral trace across the detector (e.g., Brown et al. 2001; Sing et al. 2011; Huitson et al. 2012).

We modeled the systematics and eclipse signal simultaneously as a Gaussian process (GP), following the approach of Gibson et al. (2012a, 2012b, 2013). Under the GP framework, the model likelihood takes the form of a multivariate normal distribution:

$$
p(\mathbf{f} \mid \boldsymbol{\theta})=\mathcal{N}\left(\boldsymbol{\mu}, \mathbf{K}+\sigma_{W}^{2} \mathbf{I}\right),
$$

where $\mathbf{f}=\left[f_{1}, \ldots, f_{N}\right]^{\mathrm{T}}$ are the $N=102$ measured fluxes, $\boldsymbol{\theta}$ are the model parameters, $\boldsymbol{\mu}$ is the model mean vector, $\mathbf{K}$ is the covariance matrix, $\sigma_{W}$ is the white noise level, and $\mathbf{I}$ is the identity matrix. A boxcar function was used for the eclipse signal, such that:

$$
\mu_{i}=\alpha\left(1-\delta B_{i}\right),
$$

where $\alpha$ is the constant baseline flux level, $\delta$ is the fractional flux change during eclipse, and:

$$
B_{i}= \begin{cases}0 & \text { during } 1 \text { st and 3rd orbits } \\ 1 & \text { during 2nd orbit, }\end{cases}
$$

for $i=1, \ldots, N$.

Off-diagonal entries of the covariance matrix $\mathbf{K}$ account for correlations between the measured flux and other variables that are unrelated to the eclipse signal, which we refer to as auxiliary variables (see below). We used the squared exponential kernel to populate the entries of $\mathbf{K}$, such that:

$$
K_{i j}=C^{2} \exp \left[-\sum_{d=1}^{D}\left(\frac{v_{d, i}-v_{d, j}}{L_{d}}\right)^{2}\right],
$$

where $C^{2}$ is the covariance amplitude, $v_{d, i}$ and $v_{d, j}$ are the $i$ th and $j$ th values of the $d$ th auxiliary variable, respectively, and $L_{d}$ is the correlation length scale of the $d$ th auxiliary variable. By parameterizing the covariance according to Equation (5), we effectively model the poorly understood systematics as varying 


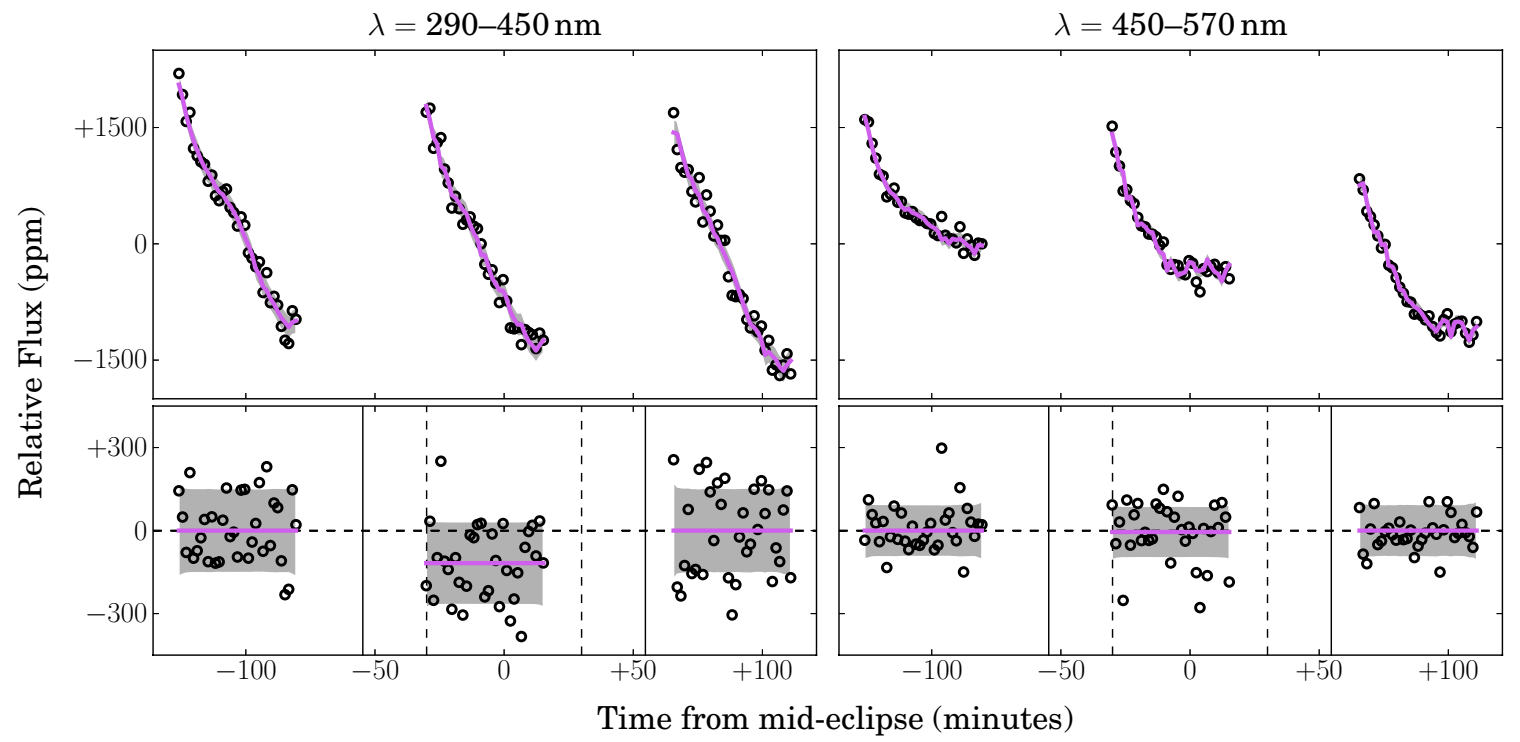

Figure 2. Top and bottom panels show, respectively, raw and corrected flux measurements (black circles) with best-fit GP model (purple lines) and per-point $1 \sigma$ uncertainties (gray shading). The $290-450 \mathrm{~nm}$ and $450-570 \mathrm{~nm}$ wavelength channels are shown on the left and right, respectively. Raw fluxes are median-subtracted and corrected fluxes show the variation about the out-of-eclipse baseline level. Bottom panels also show the start and end of ingress and egress, respectively, (solid vertical lines) and the times of full eclipse (dashed vertical lines).

(A color version of this figure is available in the online journal.)

smoothly with respect to the auxiliary variables without actually having to specify the functional form. See Gibson et al. (2012b) for more details.

For auxiliary variables, we used the orbital phase of the satellite $\phi$ and the tilt of the spectral trace on the detector $\psi$. The $\phi$-dependence accounted for the dominant systematic that repeated from orbit to orbit, while the $\psi$-dependence accounted for the smaller amplitude, higher frequency correlations as well as the longer term decrease in the flux baseline level (Figure 2). The latter was caused by an overall drift in the value of $\psi$ throughout the observations, in addition to the smaller-amplitude jitter within orbits. We also tried using the measured shifts of the spectral trace along the dispersion $x$-axis and cross-dispersion $y$-axis as additional auxiliary variables, but found their inclusion had an insignificant effect on the result. Our final parameter set therefore consisted of $\boldsymbol{\theta}=\left\{\delta, \alpha, C, L_{\phi}, L_{\psi}, \sigma_{W}\right\}$.

To marginalize Equation (2) over the space spanned by $\boldsymbol{\theta}$, we used the open source software package PyMC (Patil et al. 2010) to implement Markov Chain Monte Carlo (MCMC) sampling with the Metropolis-Hastings algorithm (Metropolis et al. 1953; Hastings 1970). We ran five chains of 120,000 steps each, where a single step consisted of cycling through the parameters and updating their values one at a time. Random step sizes were adjusted separately for each parameter to maintain step acceptance rates of $20 \%-40 \%$ throughout the chains. After discarding the first 20,000 steps as burn-in, the Gelman-Rubin values (Gelman \& Rubin 1992) were found to be well within $1 \%$ for all parameters, suggesting that the chains had converged and were well mixed.

As a check, we also modeled the systematics with different linear combinations of the auxiliary variables and used the Bayesian information criterion (Schwarz 1978) to choose between models. This has become a standard approach for analyzing HST/STIS primary transit lightcurves (e.g., Sing et al. 2011; Huitson et al. 2012). The eclipse depths inferred from these analyses were consistent with those obtained using the GP model, verifying the robustness of the results to the treatment of instrumental systematics.

\section{RESULTS}

Best-fit GP models for the two-channel binning are shown in Figure 2, with corrected lightcurves in the bottom panels. Inferred eclipse depths $\delta$ are reported in Table 1 for all wavelength channels. The median of the combined MCMC chain is quoted with uncertainties that correspond to ranges on either side containing $34 \%$ of the samples. Maximum likelihood estimates were also obtained for each parameter using the Nelder-Mead simplex algorithm (Nelder \& Mead 1965) to optimize the joint GP likelihood given by Equation (2) with respect to $\boldsymbol{\theta}$, taking the median MCMC chain values as starting points. In all cases, the binned chain values for $\delta$ had Gaussian-like distributions, and the maximum likelihood solutions were very close to the median chain values.

Table 1 also lists the values for the geometric albedo $A_{g}$, calculated using Equation (1) with the measured $\delta$ values, $\rho=$ $0.157 \pm 0.001$ (Pont et al. 2013), and $a / R_{\star}=8.863 \pm 0.020$ (Agol et al. 2010). The most striking result is that the measured albedo in the wavelength range $290-450 \mathrm{~nm}\left(A_{g}=0.40 \pm 0.12\right)$ is significantly higher than in the wavelength range $450-570 \mathrm{~nm}$ $\left(A_{g}<0.12\right)$. The broad trend of decreasing eclipse depth from shorter to longer wavelengths is also recovered from the sixchannel analysis.

In principle, variations in the brightness of the star itself, rather than the planetary eclipse, could be responsible for the measured signal. This is particularly pertinent for HD 189733, which is known to be an active $\mathrm{K}$ dwarf. However, if we assume $5000 \mathrm{~K}$ and $4200 \mathrm{~K}$ NextGen models (Hauschildt et al. 1999) for the star and spot spectra, respectively, with solar metallicity and $\log _{10} g=-4.5$, we find the flux drop in the $290-450 \mathrm{~nm}$ channel would only be $\sim 10 \%$ greater than the flux drop in the $450-570 \mathrm{~nm}$ channel. The measured difference is significantly larger than this. 
We can also estimate the characteristic amplitude of flux variations due to stellar activity using the power law index of -2.3 obtained by McQuillan et al. (2012) for the combined power spectrum of the brightest $\mathrm{K}$ dwarfs in the Q1 Kepler dataset. Scaling this to the $\sim 1 \%$ variation amplitude over $\sim 10$ day timescales appropriate for HD 189733, we obtain a corresponding amplitude of $\sim 30 \mathrm{ppm}$ in the $290-450 \mathrm{~nm}$ channel on timescales of 96 minutes (i.e., HST orbital period). This is less than half the flux change observed and slightly smaller than the uncertainty on $\delta$ due to other sources. We therefore consider it unlikely that stellar variability could account for the signal, and assume that we have indeed measured the planetary eclipse.

We are not the first to claim that the albedo of HD 189733b decreases across the visible wavelength range. Berdyugina et al. $(2008,2011)$ used polarimetry to infer albedos of $A_{g}=$ $0.61 \pm 0.12$ in the $B$-band $(390-480 \mathrm{~nm})$ and $A_{g}=0.28 \pm 0.16$ in the $V$-band (500-590 nm). Our results are systematically $\sim 2 \sigma$ lower than these values.

\section{IMPLICATIONS FOR THE ATMOSPHERE}

To first order, the reflection spectrum of a hot Jupiter at visible wavelengths will depend on whether or not there are reflective clouds in the atmosphere, and the altitude at which they become optically thick (e.g., Sudarsky et al. 2000). If scattering clouds are present and become optically thick at altitudes above the absorption wings of the $\mathrm{Na} 589 \mathrm{~nm}$ doublet and other atomic and molecular absorbers, high albedos $\left(A_{g} \sim 0.4-0.6\right)$ can be maintained throughout the visible wavelength range (e.g., Class V models of Sudarsky et al. 2000). If there are no clouds, or clouds are present but become optically thick at altitudes well below the absorption, then the albedo can be much lower. Expected values at visible wavelengths in this case will be $A_{g}<0.2$ (e.g., "irradiated" Class IV models of Sudarsky et al. 2000). In intermediate cases, if clouds are present and become optically thick at pressures comparable to the $\mathrm{Na}$ absorption wings, the albedo can be high in the blue channel relative to the red channel ${ }^{9}$ (e.g., "isolated" Class IV models of Sudarsky et al. 2000).

This list is by no means exhaustive-additional complicating factors could include the possible depletion of $\mathrm{Na}$ or the presence of condensates that absorb, rather than scatter, incident light. However, we currently have no reason to suspect that these apply to HD $189733 \mathrm{~b}$, and given the modest resolution and precision of our measurements, we restrict our discussion to the cases described above. Of these, the significantly higher albedo that is measured in the blue channel compared to the red channel is only compatible with the intermediate-altitude cloud scenario.

To investigate this possibility further, we developed a simple toy model to estimate the expected albedo spectrum. First, we took the photon deposition pressure as a function of wavelength from the pL Class model of Fortney et al. (2008, see their Figure 9) and assumed this pressure was inversely proportional to absorption. Second, we used a Rayleigh $\lambda^{-4}$ scattering profile to model the effect of reflective clouds made up of small dust grains. To simulate clouds that became optically thick at different altitudes, we varied the height of the Rayleigh profile relative to absorption in steps of two atmospheric pressure scale heights, from a high level covering the wings of the $\mathrm{Na}$ $589 \mathrm{~nm}$ doublet, down to the altitude where scattering by $\mathrm{H}_{2}$

\footnotetext{
9 In the following discussion, we refer to the $290-450 \mathrm{~nm}$ and $450-570 \mathrm{~nm}$
} wavelength ranges as the "blue" and "red" channels, respectively.

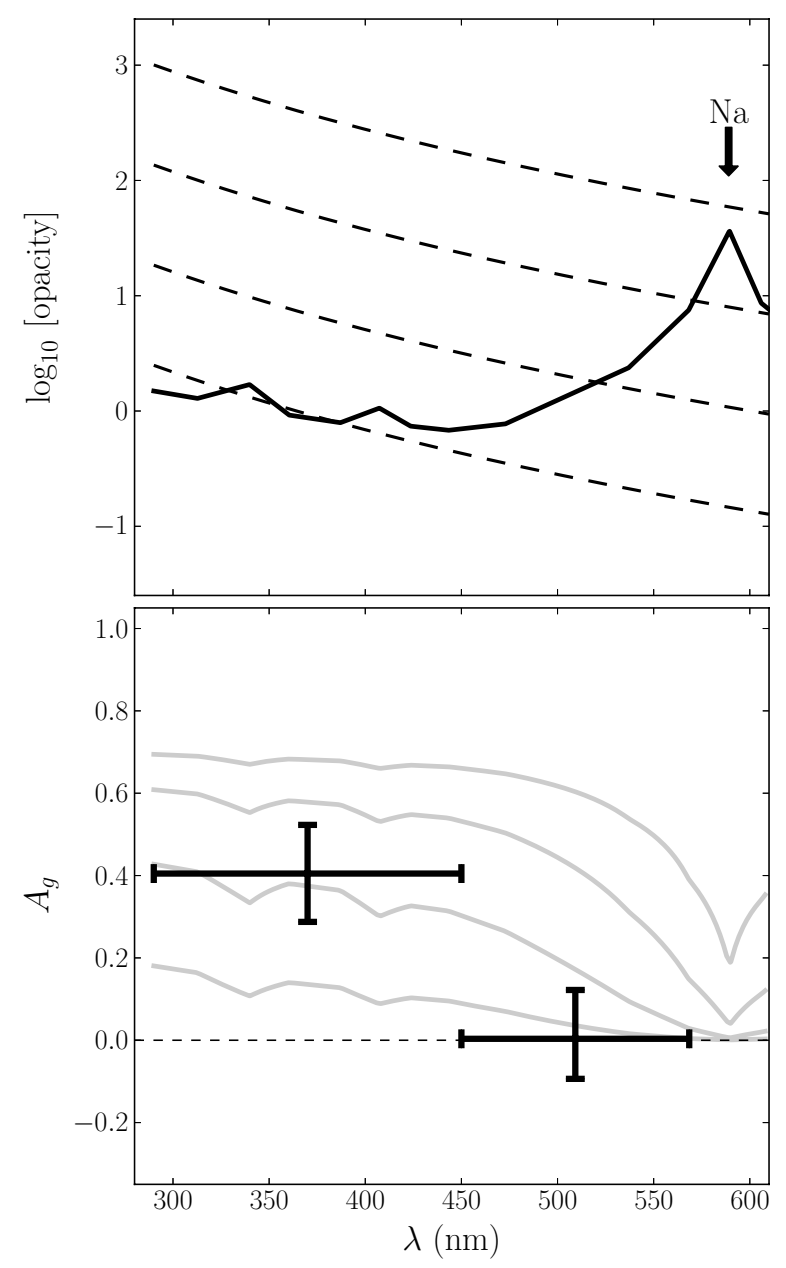

Figure 3. Top panel: relative contributions of absorption (solid line) and Rayleigh scattering (dashed lines) to the total opacity in our toy model. The scattering profiles are separated by increments of two atmospheric scale heights, to simulate clouds becoming optically thick at different altitudes. Opacity units are arbitrary as they cancel out in the toy model calculation. For reference, the base of the absorption profile is expected to correspond to pressures of 1-5 bar (e.g., Sudarsky et al. 2000; Fortney et al. 2008). Bottom panel: measured geometric albedos for the two-channel analysis (black crosses), with vertical bars indicating $1 \sigma$ uncertainties and horizontal bars indicating the extent of the corresponding wavelength channels. Toy model predictions (gray lines) are also shown for the different cloud altitudes in the top panel.

molecules becomes important. Third, with the absorption and scattering profiles defined, we calculated the albedo according to the two-stream approximation of Heng et al. (2012): $A_{g}=$ $(3 / 4)\left(1-\xi^{1 / 2}\right) /\left(1+\xi^{1 / 2}\right)$, where $\xi$ is the ratio of absorption to total opacity (absorption plus scattering) in a given passband.

The top panel of Figure 3 shows the adopted opacity profiles, split between absorption and scattering contributions, and the bottom panel shows the predicted albedo spectra with our measured values overplotted. The model with clouds becoming optically thick two scale heights above the base of the absorption profile provides a reasonable fit, although the observations favor a steeper decrease of the albedo toward longer wavelengths. Models with either gray (i.e., wavelength-independent) scattering and $\mathrm{Na}$ absorption, or Rayleigh scattering and gray absorption, were also considered but gave shallower slopes between the two channels.

An interesting question is whether or not the reflection signal is caused by the same scattering species that produces the Rayleigh profile in the transmission spectrum. Given the strong atmospheric circulation expected for hot Jupiters, this 


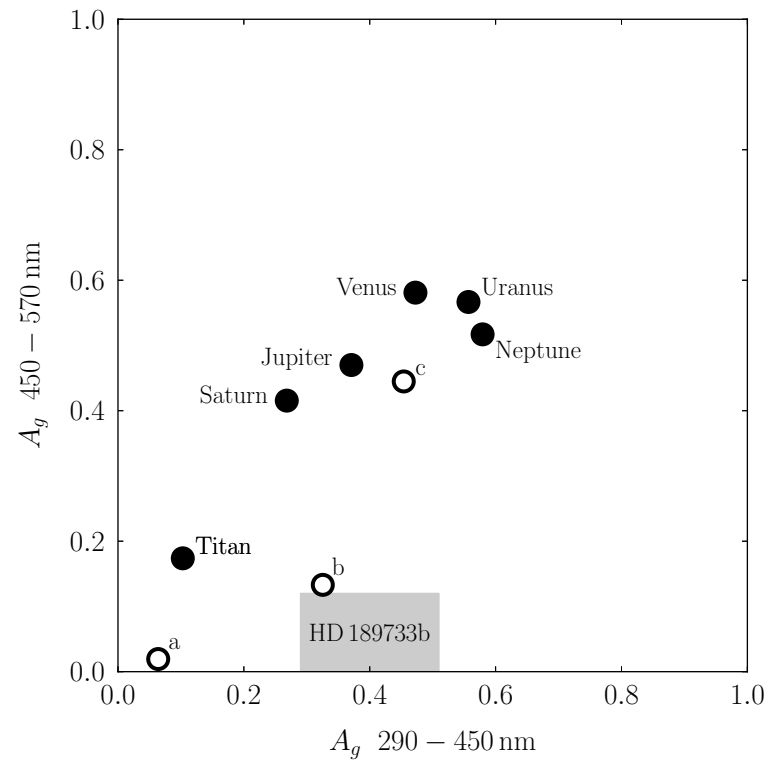

Figure 4. Color-color plot showing geometric albedos for various solar system bodies (black circles) with the $1 \sigma$ probability range for HD $189733 \mathrm{~b}$ derived in this study (gray box). Solar system albedos are from Karkoschka (1994), except for the Venus albedo which comes from Titov et al. (2007). Also shown are three models from Sudarsky et al. (2000) (open circles): (a) irradiated Class IV, (b) isolated Class IV, and (c) Class V.

seems plausible (e.g., Showman et al. 2009; Perna et al. 2012). Lecavelier Des Etangs et al. (2008) identified enstatite grains $\left(\mathrm{MgSiO}_{3}\right)$ as likely candidates, being transparent in the visible and formed of atoms abundant in hot atmospheres.

The limited precision of the current data prevents us from ruling out more intricate scenarios. For example, a high altitude tenuous haze could account for the transmission signal while being transparent at zenith geometry, with a lower, denser layer of clouds producing the reflection signal. The absorption in the red channel could also be due to an absorber other than $\mathrm{Na}$, yet to be identified. For instance, TiO is an efficient absorber in the red channel, although it is expected to have rained out of the atmosphere of HD 189733b (Fortney et al. 2008).

Our results are suggestive of a low Bond albedo $A_{B}$, which is defined as the fraction of incident starlight reflected to space at all wavelengths over all angles. This follows from the simple argument that if the $\mathrm{Na} 589 \mathrm{~nm}$ doublet is not entirely masked by clouds, the situation could be similar at wavelengths beyond $589 \mathrm{~nm}$ where theory predicts significant absorption by the $\mathrm{K}$ $770 \mathrm{~nm}$ doublet and molecules such as $\mathrm{CH}_{4}$ and $\mathrm{H}_{2} \mathrm{O}$ (e.g., Sudarsky et al. 2000). However, our observations do not provide a model-independent constraint on $A_{B}$, as only $\sim 5 \%$ and $\sim 15 \%$ of the stellar flux is emitted in the blue and red channels, respectively.

Finally, in Figure 4 we show the blue-versus-red albedos for HD 189733b and a selection of solar system bodies that also have thick atmospheres, and predicted albedos from three hot Jupiter models. Berdyugina et al. (2011) hypothesize that HD 189733b might have a reflection spectrum similar to Neptune. However, our best-fit albedos suggest that it is a deep, dark blue, quite distinct from the atmosphere colors seen in our solar system.

The results presented here demonstrate the potential of secondary eclipse observations with HST/STIS. Our albedo measurements add another piece to the growing jigsaw puzzle of empirical constraints on HD 189733b's atmosphere, through which we are gradually moving toward a more complete understanding of this exotic planet.

Based on observations made with the NASA/ESA Hubble Space Telescope, obtained at the Space Telescope Science Institute, which is operated by the Association of Universities for Research in Astronomy, Inc., under NASA contract NAS $5-26555$.

\section{REFERENCES}

Agol, E., Cowan, N. B., Knutson, H. A., et al. 2010, ApJ, 721, 1861

Alonso, R., Alapini, A., Aigrain, S., et al. 2009, A\&A, 506, 353

Alonso, R., Deeg, H. J., Kabath, P., \& Rabus, M. 2010, AJ, 139, 1481

Berdyugina, S. V., Berdyugin, A. V., Fluri, D. M., \& Piirola, V. 2008, ApJL, 673, L83

Berdyugina, S. V., Berdyugin, A. V., Fluri, D. M., \& Piirola, V. 2011, ApJL, 728, L6

Brown, T. M., Charbonneau, D., Gilliland, R. L., Noyes, R. W., \& Burrows, A. 2001, ApJ, 552, 699

Burrows, A., Ibgui, L., \& Hubeny, I. 2008, ApJ, 682, 1277

Charbonneau, D., Knutson, H. A., Barman, T., et al. 2008, ApJ, 686, 1341

Christiansen, J. L., Ballard, S., Charbonneau, D., et al. 2010, ApJ, 710, 97

Deming, D., Harrington, J., Seager, S., \& Richardson, L. J. 2006, ApJ, 644,560

Demory, B.-O., Seager, S., Madhusudhan, N., et al. 2011, ApJL, 735, L12

Désert, J.-M., Charbonneau, D., Fortney, J. J., et al. 2011, ApJS, 197, 11

Fortney, J. J., Lodders, K., Marley, M. S., \& Freedman, R. S. 2008, ApJ, 678,1419

Fortney, J. J., Shabram, M., Showman, A. P., et al. 2010, ApJ, 709, 1396

Gelman, A., \& Rubin, D. B. 1992, Stat. Sci., 7, 457

Gibson, N. P., Aigrain, S., Barstow, J. K., et al. 2013, MNRAS, 428, 3680

Gibson, N. P., Aigrain, S., Pont, F., et al. 2012a, MNRAS, 422, 753

Gibson, N. P., Aigrain, S., Roberts, S., et al. 2012b, MNRAS, 419, 2683

Grillmair, C. J., Burrows, A., Charbonneau, D., et al. 2008, Natur, 456, 767

Hastings, W. K. 1970, Biometrika, 57, 97

Hauschildt, P. H., Allard, F., \& Baron, E. 1999, ApJ, 512, 377

Heng, K., Hayek, W., Pont, F., \& Sing, D. K. 2012, MNRAS, 420, 20

Huitson, C. M., Sing, D. K., Vidal-Madjar, A., et al. 2012, MNRAS, 422, 2477

Karkoschka, E. 1994, Icar, 111, 174

Kipping, D., \& Bakos, G. 2011, ApJ, 730, 50

Kipping, D. M., \& Spiegel, D. S. 2011, MNRAS, 417, L88

Knutson, H. A., Charbonneau, D., Allen, L. E., et al. 2007, Natur, 447, 183

Knutson, H. A., Lewis, N., Fortney, J. J., et al. 2012, ApJ, 754, 22

Lecavelier Des Etangs, A., Pont, F., Vidal-Madjar, A., \& Sing, D. 2008, A\&A, 481, L83

Marley, M. S., Gelino, C., Stephens, D., Lunine, J. I., \& Freedman, R. 1999, ApJ, 513,879

McQuillan, A., Aigrain, S., \& Roberts, S. 2012, A\&A, 539, A137

Metropolis, N., Rosenbluth, A. W., Rosenbluth, M. N., \& Teller, A. H. 1953, $\mathrm{JChPh}, 21,1087$

Morris, B. M., Mandell, A. M., \& Deming, D. 2013, ApJL, 764, L22

Nelder, J. A., \& Mead, R. 1965, CompJ, 7, 308

Patil, A., Huard, D., \& Fonnesbeck, C. J. 2010, J. Stat. Softw., 35, 1

Perna, R., Heng, K., \& Pont, F. 2012, ApJ, 751, 59

Pont, F., Knutson, H., Gilliland, R. L., Moutou, C., \& Charbonneau, D. 2008, MNRAS, 385, 109

Pont, F., Sing, D. K., Gibson, N., et al. 2013, MNRAS, 432, 2917

Rowe, J. F., Matthews, J. M., Seager, S., et al. 2008, ApJ, 689, 1345

Schwarz, G. E. 1978, Ann. Stat., 6, 461

Seager, S. 2010, Exoplanet Atmospheres: Physical Processes (Princeton: Princeton Univ. Press)

Showman, A. P., Fortney, J. J., Lian, Y., et al. 2009, ApJ, 699, 564

Sing, D. K., Désert, J.-M., Lecavelier Des Etangs, A., et al. 2009, A\&A, 505,891

Sing, D. K., Pont, F., Aigrain, S., et al. 2011, MNRAS, 416, 1443

Snellen, I. A. G., de Mooij, E. J. W., \& Albrecht, S. 2009, Natur, 459, 543

Snellen, I. A. G., de Mooij, E. J. W., \& Burrows, A. 2010, A\&A, 513, A76

Sudarsky, D., Burrows, A., \& Pinto, P. 2000, ApJ, 538, 885

Titov, D. V., Bullock, M. A., Crisp, D., et al. 2007, Washington, D.C. American Geophysical Union Geophysical Monograph Series, 176, 121

Welsh, W. F., Orosz, J. A., Seager, S., et al. 2010, ApJL, 713, L145 